\title{
Stoma Site Infection
}

National Cancer Institute

\section{Source}

National Cancer Institute. Stoma Site Infection. NCI Thesaurus. Code C78629.

An infectious process affecting a surgically created opening in the surface of the body. 